\section{Propuesta de intervención: Talleres con perspectiva literaria de formación en género y derechos humanos en clave intercultural}

\author{
Intervention proposal: Workshops with a literary perspective on \\ training in gender and human rights in an intercultural key
}

\author{
Erika Silvina Bauger \\ https://orcid.org/0000-0002-6207-4203 \\ ebauger@jursoc.unlp.edu.ar \\ Facultad de Ciencias Jurídicas y Sociales | \\ UNLP I Argentina
}

\section{RESUMEN}

El proyecto parte de considerar la importancia de producir condiciones institucionales para que los derechos humanos en sentido amplio, no queden relegados a meras declaraciones retóricas, y en este sentido, procura aportar a la realización de un trabajo teórico y político pedagógico, que desde una epistemología crítica dispute las construcciones de sentido hegemónicas y avance en la transversalización, institucionalización e integralidad de este campo en la universidad pública. Los enfoques de género y derechos humanos en clave intercultural en la enseñanza práctica del derecho, vienen siendo debatidos en tanto se configuran en una vacancia en la formación docente y socio-profesional, aun cuando existe una producción teórica y política al respecto. Esta situación exige ser problematizada desde una interlocución interdisciplinaria que permita producir desplazamientos significativos que pongan en tensión enunciaciones tradicionales y aporten a la construcción de miradas inclusivas y democratizadoras de los espacios educativos.

\section{ABSTRACT}

The project starts from considering the importance of producing institutional conditions so that human rights in a broad sense, are not relegated to mere rhetorical statements, and in this sense, it seeks to contribute to the realization of a pedagogical theoretical and political work, which from an epistemology criticism dispute the hegemonic constructions of meaning and progress in the mainstreaming, institutionalization and comprehensiveness of this field in the public university. The intercultural gender and human rights approaches in the practical teaching of law have been debated as they are configured in a vacancy in teacher and socio-professional training, even when there is a theoretical and political production in this regard. This situation demands to be problematized from an interdisciplinary dialogue that allows to produce significant displacements that put in tension traditional enunciations and contribute to the construction of inclusive and democratizing views of educational spaces.
PALABRAS CLAVE talleres literarios, género, derechos humanos, educación intercultural

KEY WORDS

literary workshops, gender,

human rights, intercultural education 


\section{PRESENTACIÓN}

Los enfoques de género y derechos humanos en clave intercultural en la enseñanza práctica del derecho, vienen siendo debatidos en tanto se configuran en una vacancia en la formación docente y socio-profesional, aun cuando existe una producción teórica y política al respecto. Esta situación exige ser problematizada desde una interlocución interdisciplinaria que permita producir desplazamientos significativos que pongan en tensión enunciaciones tradicionales y aporten a la construcción de miradas inclusivas y democratizadoras de los espacios educativos.

Desde esta perspectiva y partiendo de la idea fuerza respecto a que la educación es un "derecho que da derechos", la propuesta se implementará en talleres en espacios comunitarios, con enclave barrial que faciliten el acceso a la justicia. El contenido del taller versará sobre formación en género y derechos humanos en clave intercultural.

La metodología a implementar contará con herramientas de perspectiva literaria, contenido audiovisual comunicacional, previa convocatoria abierta. El objetivo principal es generar la construcción de instrumentos para prevenir y afrontar problemáticas de género y derechos humanos desde una perspectiva crítica intercultural. Entendemos que tales conflictividades suceden en todos los ámbitos y espacios que el sujeto habita y transita, sea doméstico, laboral, escolar, comunitario barrial e intra vincular. Se abordará el logro del objetivo, a partir de procesos de enseñanza y aprendizaje que impliquen el reconocimiento e identificación de situaciones y su posterior resolución a través de la aplicación de los saberes adquiridos e integrados a la subjetividad de las/os participantes del taller. Para ello, se requiere en primer lugar la concientización acerca de las problemáticas. 
Se llevarán al taller textos literarios y material audiovisual en lenguaje claro, para la construcción colectiva de conocimiento, a partir de los disparadores que surgen de la relación con la lectura y la compresión colectiva de los textos. Se trabajarán en forma grupal las herramientas para la prevención y el abordaje de situaciones conflictivas, priorizando la participación de los concurrentes en las conclusiones, estrategias, instrumentos de análisis y acción.

Incluiremos también, la sistematización de experiencias como método para recuperar, reflexionar y difundir la propuesta desde la integralidad que supone la articulación y diálogo de los procesos de enseñanza y aprendizaje, la investigación y la extensión.

\section{RELEVANCIA Y JUSTIFICACIÓN DEL PROYECTO}

La universidad es permanentemente interpelada por las transformaciones del contexto y por las demandas sociales, y quienes trabajamos y estudiamos en ella podemos reconocernos o no en ese proceso, habilitando diferentes modos de generar respuestas en cada coyuntura. Siendo esto así, cabe preguntarse ¿cuál es la posición que la enseñanza universitaria asume frente a esas realidades? ¿Cómo repensar la docencia y la extensión en escenarios inéditos y profundamente desiguales como el que actualmente se vive por efecto de la pandemia?, ¿qué papel juegan los derechos humanos y particularmente las dimensiones de género cuando la apuesta es formar profesionales críticos y comprometidos con los problemas de sus pueblos?

¿cuál es la posición que la enseñanza universitaria asume frente a esas realidades? ¿Cómo repensar la docencia y la extensión en escenarios inéditos y profundamente desiguales como el que actualmente se vive por efecto de la pandemia?, ¿qué papel juegan los derechos humanos y particularmente las dimensiones de género cuando la apuesta es formar profesionales críticos y comprometidos con los problemas de sus pueblos?

Incorporar estos enfoques posibilita descubrir y fortalecer espacios de acción político-pedagógica indispensables para procesar las demandas de la sociedad y avanzar hacia propuestas educativas que aborden la complejidad de los cambios, alojen lo nuevo y construyan condiciones de igualdad que reconozcan la diversidad y la diferencia. $\mathrm{O}$, dicho de otro modo, referenciar la educación superior como derecho que da derechos, implica visibilizar el papel socio-histórico y político que la universidad juega en relación a la producción y disputa de sentidos sobre un inter-venir y un por-venir. 
La importancia del proyecto estriba en pensar a la extensión universitaria como actividad que permite la construcción de saberes en consonancia con prácticas en territorios supervisadas, bajo modalidades institucionalmente articuladas y acompañadas. En este proceso no podemos circunscribir esta construcción y responsabilidad exclusivamente en los/as sujetos/as destinatarias, ya que la interculturalidad requiere la pluralidad de voces y el compromiso de toda la sociedad.

Este tipo de prácticas permite vincular saberes y contenidos a situaciones concretas con poblaciones y sujetxs con Ixs que trabajar codo a codo, lo que implica vincular en esas situaciones concretas, teoría y práctica, y un posicionamiento ético profesional. La extensión al volverse crítica e integral presenta un desafío didáctico y político-pedagógico con el que pensar cómo transmitir saberes, pero también recibirlos de los espacios y personas con quienes trabajamos.

La extensión aparece entonces, como el espacio que permite trabajar y vincular saberes y contextos de aplicación y aprendizaje. Pensamos que, a nivel social, la universidad es un actor político fundamental para dar respuesta y acompañamiento a procesos que se dan en los territorios donde se ubica. Al ser uno de sus pilares, la extensión es pensada y revisada tanto institucionalmente como socialmente en cada momento de su implementación, pero muchas veces ha quedado relegada o incluso ha sufrido los embates de las culturas institucionales que la dejan en un lugar de menor importancia con respecto a la docencia o a la investigación. Ésta, también, es una motivación a la hora de pensar que la extensión puede ser una gran herramienta de intervención a la hora de formar profesionales comprometidxs con sus contextos sociales.

En particular, y siguiendo este hilo, pensamos en la posibilidad de planificar un proyecto para llevarlo a cabo en los barrios en los que funcionan los consultorios jurídicos gratuitos y particularmente una experiencia de extensión innovadora en el barrio donde habita la comunidad Qom de La Plata. En la primera intervención, el desafío será volver a estos barrios desde otro lugar, retomando problemas que se han trabajado en reuniones específicas con referentes y actores barriales, para armar agendas de problemas en co-gestión. Y en el caso de la comunidad Qom, generar mediante una hermenéutica pluritópica de la dignidad humana que permita enriquecer este concepto y fundamentar los derechos humanos desde una concepción intercultural y pluralista.

En función de esto, la propuesta se centra en desarrollar talleres literarios que funcionen como disparadores mediante una metodología pre dialógica, que permita abordar de una manera lo más agradable posible, el aprendizaje de derechos que tienen las personas en sus contextos particulares, para enfrentar discriminaciones y violencias y lograr un empoderamiento personal. 
la propuesta se centra en desarrollar talleres literarios que funcionen como disparadores mediante una metodología pre dialógica, que permita abordar de una manera lo más agradable posible, el aprendizaje de derechos que tienen las personas en sus contextos particulares, para enfrentar discriminaciones y violencias y lograr un empoderamiento personal.

Hoy, en este contexto de pandemia, el desafío se vuelve doble, y nos interpela a pensar y actuar en torno a cómo elaborar propuestas que nos permitan trabajar estas temáticas con el cuidado y la responsabilidad que la problemática impone en territorios del Gran La Plata, y cómo sostener a un equipo que quiere capacitarse en estas temáticas, que creemos, pueden generar grandes reflexiones en contextos de virtualidad y encuentros reducidos.

\section{OBJETIVOS Y RESULTADOS}

3.1. Objetivo General: Crear un espacio de construcción colectiva de saberes, donde se genere conocimiento de carácter intercultural, con la participación de la población en convocatoria abierta, en la modalidad de talleres con perspectiva literaria. El objetivo, integra la posibilidad de crear herramientas de identificación y resolución de situaciones vinculadas a problemáticas de género y derechos humanos, que atraviesan las dimensiones del cotidiano, ámbito doméstico, comunitario, laboral, escolar e intra vincular.

Objetivos Específicos: 1.-Implementar la modalidad de talleres barriales y la afluencia de concurrentes, mediante una convocatoria abierta. 2.-Lograr convocatorias exitosas en concurrencia, para lo cual se necesitará una estrategia de difusión adecuada. 3.-Participación grupal y horizontal en la construcción de conocimientos. Crear un espacio de discusión y debate sobre la importancia de la perspectiva de género y derechos humanos y su potencial transformador. 4.-Generar conciencia y posibilidad de identificar problemáticas vinculadas a la temática de género y derechos humanos en clave intercultural. 5.-Proveer herramientas de intervención y deconstrucción de dichas problemáticas, tanto individuales como colectivas. 6.-Lograr empoderar a Ixs participantes del taller, mediante la construcción colectiva de herramientas de diálogo y acción que surjan en el espacio. 7.-Promover un empoderamiento personal y colectivo que actúe de forma preventiva en los conflictos vinculados al género y los derechos humanos. 8.-Transmisión a la comunidad y a la universidad de los saberes construidos grupalmente. 9.-Generar un espacio de intercambio y produc- 
ción tendiente a identificar recursos y dispositivos que favorezcan su apropiación crítica por parte de les estudiantes y que se concrete en un trabajo de investigación aplicada.10.-Sistematizar la praxis extensionista, lo que nos permitirá elaborar una propuesta categórica desde lo académico para los procesos de interpelación de una institución en contexto, en temáticas relativas al género y a los derechos humanos de las mujeres y otras identidades no hegemónicas.

\subsection{Resultados esperados}

La actividad de extensión tiene por objetivo principal, hacer una devolución a la comunidad de aquello aprendido como saber en la Universidad. En los talleres se intentará lograr como resultado específico, la generación de una "ecología de saberes" -al decir de Boaventura de Sousa Santos (2014)- y democratizar la enseñanza. Lograr que en el espacio dialoguen el saber científico, el saber laico, el saber popular, el saber indígena, el saber de las poblaciones urbanas marginadas. La construcción colectiva de herramientas desde esta ecología, permitirá la intervención para tratar las temáticas vinculadas al género y a los derechos humanos, en el saber de qué dicha conflictiva es transversal a todos los ámbitos donde una persona se desenvuelve, doméstico, escolar, laboral, intra vincular.

\section{La profundización de los procesos de extensión universitaria no son solamente un camino adecuado sino, más bien, es el co- mienzo del camino que debemos transitar hacia el espacio de integralidad, donde simplemente la docencia, investigación y extensión sean cotidianas en la formación en contexto y donde los procesos de extensión alimenten a las líneas de enseñanza y la investigación, y viceversa.}

\subsection{Indicadores de progreso y logro}

Ex ante: antes de la puesta en práctica de la propuesta, se realizaron entrevistas con referentes de los barrios. Se analizó si los componentes de la innovación son coherentes y sustentables. Se realizó el análisis documental (textos, materiales audiovisuales, etc.) para la puesta en práctica de la actividad.

Durante: se realizará una evaluación del proceso que permita la valoración colectiva acerca de la experiencia que llevan adelante los sujetxs, así como la retroalimentación del equipo de trabajo con el fin de ajustar el desarrollo de la propuesta formativa. Ello supondrá al finalizar cada encuentro, la valoración colectiva del desarrollo de la jornada teniendo especialmente en cuenta el trabajo realizado, los aportes significativos que les proveyera tanto el intercambio con 
los coordinadores del taller como con las/os participantes, como así también propuestas para el mejoramiento de las futuras acciones. Se identificarán efectos no previstos, la respuesta de las/os participantes y se realizarán los informes del proceso.

Ex post: la evaluación al final del proceso se centrará en el análisis de resultados, errores, imprevistos, asistentes y retroalimentación de y con las/os participantes. Análisis de este tipo de evaluación a través del tiempo y de los resultados para la resolución que dio lugar a la innovación.

\section{METODOLOGÍA}

La metodología consistirá en la implementación de talleres, de dinámicas dialógicas, horizontales y participativas, en donde se generen herramientas para tratar conflictividades vinculadas al género y a los derechos humanos en clave intercultural.

Se utilizará la metodología de la sistematización de experiencias, a fin de lograr un registro de la construcción colectiva que surja de los talleres, como camino que debemos transitar hacia el espacio de integralidad, donde simplemente la docencia, la investigación y la extensión sean cotidianas en la formación en contexto y donde los procesos de extensión alimenten a las líneas de enseñanza y la investigación, y viceversa. Porque, en este firme desafío, encontraremos la democratización del conocimiento generado en situaciones concretas de vida de la comunidad. Que la falta de Pueblo en nuestra Universidad y en nuestra Facultad no se naturalice, porque estaremos deslegitimizando una institución que amamos y como ciudadanas y ciudadanos contenemos.

La modalidad de taller se propone con la finalidad de favorecer la articulación continua entre teoría y práctica, promoviendo la revisión de ambas en su vinculación. Partiendo de la idea fuerza respecto a que la extensión no es a priori, sino que da forma a las necesidades del grupo al cual se dirige en un contexto económico y social específico, la extensión será una "intención" de internalizar la agenda de discusión social como directiva, desde un enfoque interdisciplinario que es como creemos se abordan los problemas de las personas. Intención de hacer algo en el sentido de internalizar, y traer para adentro de la Facultad esa agenda de discusión social (Dagnino, 2008). En esta línea de trabajo se pueden señalar algunas estrategias que se utilizarán, configurando sólo una hipótesis:

-Actividades de ubicación y apertura al tema propuesto. Presentación de textos literarios, obras de teatro o cualquier material audiovisual en lenguaje claro, como disparador para presentar los problemas centrales que se pretenden trabajar. Ubicación de las perspectivas teóricas, expectativas y saberes que se recuperan para abordarlo, situando los aportes de la bibliografía. 
-Estrategias de problematización de los temas propuestos en función de relevar las visiones y perspectivas de les participantes.

-Instancias de trabajo en subgrupos para el abordaje de situaciones problemáticas.

Se solicitará tener a disposición un Aula virtual (cátedra virtual) para proyectar cada encuentro en forma sincrónica y facilitar la participación de estudiantes que no puedan trasladarse al barrio.

\section{UNIDAD ACADÉMICA Y EJECUTORA. IDENTIFICACIÓN DE LAS/OS DESTINATARIAS/OS}

Como unidad académica y ejecutora se propone la intervención de la Secretaría de Extensión de Facultad de Ciencias Jurídicas y Sociales de la UNLP.

La convocatoria es abierta y dirigida a tres sujetos/as: 1) Personas que habitan en el barrio perteneciente a la comunidad Qom de la Plata. 2) Personas que habitan en las inmediaciones del consultorio jurídico gratuito de Villa Elisa en la (Casa del Niño "Arco Iris") perteneciente al programa "El derecho se tiene cuando se conoce y se ejerce" de la Secretaría de Extensión de la Facultad de Ciencias Jurídicas y Sociales de la UNLP.3) Estudiantes de grado de la carrera de Abogacía que podrán acreditar mediante un trabajo final que refleje una producción académica, su participación en los talleres.

Los talleres se dirigirán principalmente a personas mayores de edad, pero se prevé la realización de encuentros específicos relacionados con temas de niñez y adolescencias, tales como talleres de enseñanza y aprendizaje de Educación Sexual Integral (ESI).

Como uno de los requisitos para la obtención de su título según el plan de estudio vigente, el alumno elabora un trabajo de investigación. De acuerdo al Reglamento de los Seminarios de Grado, existen tres modalidades de emprendimiento: a) seminarios cursados interdisciplinarios; b) seminarios por pasantía y c) seminarios de investigación aplicada (libre). Dentro de esta última modalidad, se ubicaría la experiencia del estudiante que participe en los talleres. Entendemos aquí, por investigación aplicada en el ámbito del derecho la que se realiza utilizando las técnicas de investigación llamadas humanísticas, sociológicas, realistas, "prácticas" o "materiales", por oposición a las que utiliza la investigación fundamental: técnicas, teóricas, dogmáticas, "conceptuales" o "formales".

\section{LOCALIZACIÓN GEOGRÁFICA}

Se propone la localización de dos talleres como experiencias piloto.

1) Un taller innovador con la Comunidad Nam Qom de La Plata en 
155. Comprende un área de veintidós manzanas, subdivididas en 540 lotes de los cuales 36 pertenecen a la comunidad Nam Qom.

2) Un taller donde funciona actualmente el consultorio jurídico gratuito de Villa Elisa en la calle 423 bis entre 12 y 13 (Casa del Niño "Arco Iris"), perteneciente al programa "El derecho se tiene cuando se conoce y se ejerce" de la Secretaría de Extensión de la Facultad de Ciencias Jurídicas y Sociales de la UNLP.

En el contexto actual de clases no presenciales, analizamos la incorporación de herramientas tecnológicas para la participación de los/ as estudiantes en los talleres a través de las actividades que provee un entorno virtual de enseñanza y aprendizaje como son las cátedras virtuales. Desde esa plataforma, se organizarán los encuentros sincrónicos a través de la organización de reuniones por webex meeting, zoom, google classroom o jitsi meet y la utilización de un canal de YouTube para encuentros asincrónicos y cualquier otra herramienta virtual que permita tender puentes entre docentes y estudiantes hacia un proyecto, hacia un mañana, hacia un aprendizaje.

En el contexto actual de clases no presenciales, analizamos la incorporación de herramientas tecnológicas para la participación de los/as estudiantes en los talleres a través de las actividades que provee un entorno virtual de enseñanza y aprendizaje como son las cátedras virtuales.

\section{RESPONSABLE/S DEL PROYECTO, EQUIPO DE TRABAJO Y ORGA- NIZACIONES CO-PARTÍCIPES. EQUIPAMIENTO DISPONIBLE}

La propuesta es que sea interdisciplinar, horizontal entre las/os integrantes del equipo de trabajo que participen de los encuentros. Proponemos que los cargos sean rotativos en cada cuatrimestre del período lectivo.

La convocatoria para la integración del equipo permanecerá abierta conforme a las necesidades del espacio de intervención a docentes, extensionistas e investigadoras de distintas disciplinas.

Como copartícipes de la organización del trabajo en el territorio se propone la participación de la Comunidad Nam Qom de La Plata y de la Casa del Niño "Arco iris".

Para llevar a cabo el proyecto contaremos con dos espacios físicos: 1) El salón donde funciona actualmente el consultorio jurídico gratuito de Villa Elisa en la calle 423 bis entre 12 y 13 (Casa del Niño "Arco Iris"), perteneciente al programa "El derecho se tiene cuando se conoce y se ejerce" de la Secretaría de Extensión de la Facultad de Ciencias 
Jurídicas y Sociales de la UNLP. 2) El salón comunitario de usos múltiples perteneciente a la comunidad Nam Qom de La Plata, en el barrio Malvinas que comprende las calles 32 hasta 36 y 149 hasta 155.

Ambos espacios cuentan con los servicios básicos y mobiliario para trabajar en los talleres.

También necesitaremos para trabajar en los dos espacios: 2 computadoras, 2 proyectores y 2 cámaras fotográficas, que solicitaremos a la Facultad de Ciencias Jurídicas y Sociales de la UNLP.

\section{ACTIVIDADES, DURACIÓN DEL PROYECTO Y CRONOGRAMA}

Se planea el siguiente cronograma de actividades:

Primer año

-Búsqueda y exploración bibliográfica. Revisión de antecedentes y metodología. Organización del trabajo de campo. Conformación de los equipos de trabajo. Acercamiento al campo.

-Difusión y convocatoria abierta en el ámbito barrial donde se desarrollará el taller, con la colaboración de la entidad que aporte el espacio físico para la actividad.

-Difusión y convocatoria abierta desde la Facultad de Ciencias Jurídicas y Sociales UNLP.

-Encuentro con los referentes barriales que colaboran en el desarrollo de la actividad. Se realizará un conversatorio con las personas que estén a cargo del lugar donde se realicen los talleres, presidentas de las ONG que aporten el espacio, trabajadores de dichos espacios, referentes barriales que quieran participar, etc.

-Generación de espacios de diálogo con los/as directores de los consultorios jurídicos gratuitos, a fin de estar en permanente contacto con las problemáticas de dicho espacio.

-Reuniones del equipo interdisciplinario de trabajo para planificar las estrategias, métodos materiales literarios y audiovisuales para trabajar en los talleres.

-Encuentros en el territorio en la modalidad de taller. El equipo organizará la actividad con el objetivo de la participación activa de las personas concurrentes.

-Actividades dentro del taller: se trabajará con material literario, proyección audiovisual, podcast y bibliografía jurídica. Este material actuará como disparador de cuestiones vinculadas a la problemática 
de género y derechos humanos en clave intercultural. Los temas serán tratados desde aquellas conflictividades que no se presentan como explícitas, pero subyacen el cotidiano.

-En forma simultánea a la realización del taller, se llevará adelante la construcción de registros y herramientas metodológicas que nos permitan sistematizar las experiencias que se den en el espacio.

- Análisis de resultados. Evaluaciones durante y ex post de las experiencias.

- Elaboración de un informe parcial interno de evaluación del proyecto.

- A los fines de garantizar la transparencia y el acceso a la información pública, se elaborará y presentará un informe final institucional.

- Se orientará a las/os estudiantes en la realización del trabajo final integrador de investigación para acreditarlo como Seminario de grado.

- Presentaciones en Congresos y Jornadas para la adecuada difusión del proyecto.

\section{Segundo Año}

-Continuidad de encuentros con los referentes barriales que colaboran en el desarrollo de la actividad. Se realizará un conversatorio con las personas que estén a cargo del lugar donde se realicen los talleres, presidentas de las ONG que aporten el espacio, trabajadores de dichos espacios, referentes barriales que quieran participar, etc.

-Continuidad de difusión y convocatoria abierta en el ámbito barrial donde se desarrollará el taller, con la colaboración de la entidad que aporte el espacio físico para la actividad.

-Generación de espacios de diálogo con los directores de los consultorios jurídicos gratuitos, a fin de estar en permanente contacto con las problemáticas de dicho espacio.

-Reuniones del equipo interdisciplinario de trabajo para planificar las estrategias, métodos materiales literarios y audiovisuales para trabajar en los talleres.

-Encuentros en el territorio en la modalidad de taller. El equipo organizará la actividad con el objetivo de la participación activa de las personas concurrentes.

-Actividades dentro del taller: se trabajará con material literario, 
proyección audiovisual, podcast y bibliografía jurídica. Este material actuará como disparador de cuestiones vinculadas a la problemática de género y derechos humanos en clave intercultural. Los temas serán tratados desde aquellas conflictividades que no se presentan como explícitas, pero subyacen el cotidiano.

-En forma simultánea a la realización del taller, se llevará adelante la construcción de registros y herramientas metodológicas que nos permitan sistematizar las experiencias que se den en el espacio.

-Análisis de resultados. Evaluaciones durante y ex post de las experiencias.

- Se orientará a las/os estudiantes en la realización del trabajo final integrador de investigación para acreditarlo como Seminario de grado.

-Presentaciones en Congresos y Jornadas para la adecuada difusión del proyecto.

-Elaboración de un informe parcial interno de evaluación del proyecto.

-A los fines de garantizar la transparencia y el acceso a la información pública, se elaborará y presentará un informe final institucional.

\section{Tercer Año}

-Continuidad de encuentros con los referentes barriales que colaboran en el desarrollo de la actividad. Se realizará un conversatorio con las personas que estén a cargo del lugar donde se realicen los taIleres, presidentas de las ONG que aporten el espacio, trabajadores de dichos espacios, referentes barriales que quieran participar, etc.

-Continuidad de difusión y convocatoria abierta en el ámbito barrial donde se desarrollará el taller, con la colaboración de la entidad que aporte el espacio físico para la actividad.

-Generación de espacios de diálogo con los/as directores de los consultorios jurídicos gratuitos, a fin de estar en permanente contacto con las problemáticas de dicho espacio.

-Reuniones del equipo interdisciplinario de trabajo para planificar las estrategias, métodos materiales literarios y audiovisuales para trabajar en los talleres.

-Encuentros en el territorio en la modalidad de taller. El equipo organizará la actividad con el objetivo de la participación activa de las personas concurrentes. 
-Actividades dentro del taller: se trabajará con material literario, proyección audiovisual, podcast y bibliografía jurídica. Este material actuará como disparador de cuestiones vinculadas a la problemática de género y derechos humanos en clave intercultural. Los temas serán tratados desde aquellas conflictividades que no se presentan como explícitas, pero subyacen el cotidiano.

-En forma simultánea a la realización del taller, se llevará adelante la construcción de registros y herramientas metodológicas que nos permitan sistematizar las experiencias que se den en el espacio.

-Análisis de resultados. Evaluaciones durante y ex post experiencias.

-Se orientará a les estudiantes en la realización del trabajo final integrador de investigación para acreditarlo como Seminario de grado.

-Presentaciones en Congresos y Jornadas para la adecuada difusión del proyecto.

-Elaboración de un informe parcial interno de evaluación del proyecto.

-A los fines de garantizar la transparencia y el acceso a la información pública, se elaborará y presentará un informe final institucional.

-Organización de Jornadas de Intercambio y transferencia de conocimiento.

-Publicación de resultados en revistas de la Universidad Nacional de La Plata.

-Publicación en formato libro del trabajo de sistematización de la experiencia de extensión.

\section{AUTOEVALUACIÓN Y SOSTENIBILIDAD/ REPLICABILIDAD DEL PROYECTO}

Los méritos principales de este proyecto radican en: 1.- Construir saberes en territorio en forma participativa, horizontal, plural e interdisciplinaria, donde se produzcan nuevos conocimientos y herramientas de acción para tratar problemáticas en forma preventiva, en cualquier ámbito donde las personas habiten y transiten. 2.- Otra cualidad del proyecto, está dada por la riqueza de contenido de los encuentros territoriales. Esta posibilidad surge por la transversalidad que implica tratar temáticas vinculadas al género y derechos humanos en clave intercultural. 
Las experiencias del presente proyecto, aspiran a ser replicadas en los distintos espacios donde actualmente funcionan los consultorios jurídicos gratuitos incluidos en el programa "El derecho se tiene cuando se conoce y se ejerce", perteneciente a la Secretaría de Extensión de la Facultad de Ciencias Jurídicas y Sociales de la UNLP. Entendemos que estos talleres tienen como efecto, prevenir y reducir el nivel de conflictividad y así evitar la multiplicación de casos judiciales, repercutiendo en la menor sobrecarga de trabajo en los consultorios jurídicos gratuitos.

\section{Entendemos que estos talleres tienen como efecto, prevenir y reducir el nivel de conflictividad y así evitar la multiplicación de casos judiciales, repercutiendo en la menor sobrecarga de trabajo en los consultorios jurídicos gratuitos.}

Asimismo, y teniendo en cuenta la ausencia de un consultorio jurídico gratuito que garantice el acceso a la justicia de grupos vulnerables en el barrio de la Comunidad Qom de La Plata -que se propone para la intervención-, de las experiencias que se vivan en el presente proyecto, se evaluará la necesidad o no de su instalación. 


\section{BIBLIOGRAFIA}

La bibliografía abarcará textos teóricos académicos que formarán el marco teórico y político pedagógico del equipo interdisciplinario de trabajo y que se dividirán en dos ejes (Eje 1: Derechos Humanos en clave intercultural y Eje 2: Género y perspectiva de género); y textos literarios y materiales audiovisuales para trabajar el taller.

\section{Marco teórico y político pedagógico:}

\section{Eje 1: Derechos Humanos en clave intercultural}

Abramovich, Víctor y Courtis, Christian (2002). Cap. 1 "La estructura de los derechos sociales y el problema de su exigibilidad". En Los derechos sociales como derechos exigibles. Madrid: Editorial Trotta, S.A. [en línea] http://ijdh. unla.edu.ar/advf/documentos/2017/02/589352927ac71.pdf

Aguirre, Eduardo Luis (2020). Conjurando el hervidero espantoso: filosofía del derecho de nuestra América. 1a ed. - La Plata: Arte editorial Servicop.

Bobbio, Norberto (1991). "Introducción" y "Presente y provenir de los derechos humanos". En El tiempo de los derechos, (pp. 13-24 y 63-84). Madrid: Editorial Sistema.

Colombato, Lucía Carolina (2017). "Tres dimensiones y tres principios rectores del derecho humano a los patrimonios culturales". Revista de Derechos Humanos y Estudios Sociales, Año IX, Nro. 17, Enero-junio 2017, pp. 177-203.

Cruz, Verónica (2020). “El derecho a la educación superior como proceso histórico, abierto y disputado". En Parte V, libro Investigar en Cuerpo, Arte y Comunicación. Perspectivas e intersecciones en la producción de conocimiento (pp. 259 -272). Compilado por Martín Scarnatto y Fabián Amilcar De Marziani. La Plata: Editorial Teseo Press.

Declaración Universal de los Derechos Humanos, 1948. Disponible en http://www.un.org/es/documents/udhr/index_print.shtml

De Sousa Santos, Boaventura (2014). Derechos humanos, Democracia y Desarrollo. Bogotá: Colección Dejusticia. Bogotá. 1era edición. En http:// www.dejusticia.org

ONU (2014). Programa Mundial de Educación en Derechos Humanos.

Rodino, Ana M. (2014). “Pensar la educación en derechos humanos como política pública". Revista de ciencias sociales Segunda Época, № 25 pp. 129 a 139.

Salvioli, Fabián (2009). La universidad y la educación en el siglo XXI. Los derechos humanos como pilares de la nueva Reforma Universitaria. Cap. 3 "Universidad y Derechos Humanos". IIDH. 
Segato, Rita (2012) Racismo, discriminación y acciones afirmativas. Herramientas conceptuales. Observatório da Jurisdição Constitucional. Ano 5, 2011/2012. Disponible en http://ijdh.unla.edu.ar/advf/documentos/2018/03/5aba58eda4b63.pdf

\section{Otros recursos y materiales audiovisuales}

Ministerio de Justicia y Derechos Humanos de la Nación (2019). Constitución argentina en lectura fácil. Argentina: Ediciones SAIJ de la Dirección Nacional del Sistema Argentino de Información Jurídica. 3ra. edición corregida y ampliada: junio de 2019.

Ministerio de Justicia y Derechos Humanos de la Nación (2018). Convención interamericana sobre la protección de los derechos humanos de las personas mayores: en lectura fácil. - 1a ed. ilustrada. Ciudad Autónoma de Buenos Aires: Ediciones SAIJ.

Intervención del Dr. Fabián Salvioli y de la Dra. Mónica Pinto en Derechos Humanos PBA: Disponible en https://www.youtube.com/watch?v=vGFr1 mSgwBQ

Entrevista: "Educación en y para los derechos humanos" - Ana María Rodino. Derechos Humanos PBA: Disponible en https://www.youtube.com/watch?v=vGFr1mSgwBQ

Materiales elaborados en el macro de la Cátedra UNESCO Educación Superior y Pueblos Indígenas y Afrodescendientes en América Latina de la Universidad Nacional de Tres de Febrero (UNTREF), a través de su Iniciativa para la Erradicación del Racismo en la Educación Superior, orientadas a promover reflexiones y debates sobre las múltiples formas de racismo que aún persisten en los sistemas de Educación Superior, y contribuir a su erradicación. Disponibles en http:// unesco.untref.edu.ar/

Derechos de los pueblos indígenas. Dictámenes del Ministerio Público Fiscal ante la Corte Suprema de Justicia de la Nación (2012 - 2017). Dirección General de Derechos Humanos Edición: Dirección de Relaciones Institucionales. Publicación: Enero 2018.

\section{Eje 2: Género y perspectiva de género}

Barrancos, Dora (2019). Devenir feminista, Una trayectoria político intelectual. Compilado por Ana Laura Martín; Adriana María Valobra. I Edición. Ciudad Autónoma de Buenos Aires: CLACSO. Editorial de la Facultad de Filosofía y Letras.

Barrancos, Dora (2014). "Géneros y sexualidades disidentes en la Argentina: de la agencia por derechos a la legislación positiva". En Cuadernos Intercambio sobre Centroamérica y el Caribe, Vol. 11, No. 2 Julio-Diciembre, 2014, 17-46. 
la enseñanza del derecho". Derechos En Acción, 11(11), 277. Disponible en https://doi.org/10.24215/25251678e277

Bodelón, Encarna (2009). "Feminismo y derecho: mujeres que van más allá de lo jurídico". En: Gemma Nicolás y Encarna Bodelón (comps.), Género y dominación. Críticas feministas del derecho y el poder. Barcelona: Antrhopos.

Bonino, L. (1995). Desvelando los micromachismos en la vida conyugal. En J. Corsi (Eds.), Violencia masculina en la pareja. Una aproximación al diagnóstico y a los modelos de intervención (pp. 191-208). Buenos Aires: Paidós.

Carosio, Alba (2017). Perspectivas Feministas para ampliar horizontes del pensamiento crítico latinoamericano. En Feminismos, pensamiento crítico y propuestas alternativas en América Latina / Alba Carosio ... [et al.]; coordinación general de Montserrat Sagot.- 1a ed. - Ciudad Autónoma de Buenos Aires: CLACSO. Libro digital, PDF - (Grupos de trabajo / Atilio Alberto Boron).

Colanzi, Irma, Femenías, María Luisa y Seone, Viviana (compiladoras) (2016). "Los Ríos Subterráneos", Volumen V, Violencia contra las mujeres, la subversión de los discursos. Rosario: Prohistoria ediciones.

Dagnino, Renato (2008). Empezando por la extensión universitaria... Conferencia presentada en II Seminario Iberoamericano de Ciencia y Tecnología para el Hábitat Popular. Córdoba, Argentina, 2007. Disponible en http://www.proglocode.unam.mx/sites/proglocode.unam.mx/files/Dagnino\%2C\%20Empezando\%20por\%20la\%20Extensi\%C3\%B3n\%20Universitaria.pdf

Falquet, Jules (2017). "La combinatoria straight: Raza, clase, sexo y economía política: análisis feministas materialistas y decoloniales". Descentrada. Revista interdisciplinaria de feminismos y género, 1 (1), e005. En Memoria Académica. Disponible en http://www.memoria.fahce.unlp.edu.ar/art_revistas/ pr.7718/pr.7718.pdf

Fernández, Josefina (2003). Los cuerpos del feminismo. En Maffía, Diana (ed.) Sexualidades Migrantes, Género y Transgénero. Editorial Feminaria: Buenos Aires, Argentina 2003, (pp. 138-154).

Grossman, Claudio y Shalleck, Ann (1999). “Programa de Derecho de la Mujer y Derecho Internacional". En: Facio, Alda y Fries, Lorena. Género y Derecho (pp. 9 - 12). Santiago de Chile: LOM.

Lagarde, Marcela (1996) "El género", fragmento literal: "La perspectiva de género'. En Género y feminismo. Desarrollo humano y democracia (pp. 13-38). España: Ed. horas y HORAS.

Mattio, Eduardo (2012) ¿De qué hablamos cuando hablamos de género? Una introducción conceptual. En: Morán Faúndes, J.; Sgró Ruata, M. C. y Vaggione, J. M. (edits.). Sexualidades, desigualdades y derechos: reflexiones 
en torno a los derechos sexuales y reproductivos. Córdoba, Argentina: Ciencia, Derecho y Sociedad Editorial.

Olsen, Frances (1990). "El sexo del derecho" en Ruiz, Alicia. Identidad femenina y discurso jurídico. Biblos. Buenos Aires.

Pitch, Tamar (2010). "Sexo y género de y en el derecho: el feminismo jurídico". En Un panorama de filosofía jurídica y política: (50 años de "Anales de la Cátedra Francisco Suárez") (pp. 435-460). Italia: Anales de la Cátedra Francisco Suárez.

Ruiz, Alicia (2000). Identidad femenina y discurso jurídico. Buenos Aires: Biblos.

Zúñiga Añazco, Yanira (2009). “La'generización' de la ciudadanía. Apuntes sobre el rol de la diferencia sexual en el pensamiento feminista". Revista de Derecho Universidad Austral de Chile, V. XXII/ № 2 (diciembre 2009), ps. 39-64.

Textos literarios y materiales audiovisuales para el Taller(estos son estimativos y se presentan sólo como una hipótesis)

Bodecker, N.M. y Blegvad, Erik (2001). Corre, corre, Mary, corre. Moix, Ana María (trad.). Barcelona: Lumen.

Brown, Anthony (1991) El libro de los cerdos. Disponible en https://es.slideshare.net/marcelobosa/el-libro-de-los-cerdos-anthony-brown

Caitlin Moran (2013). Cómo ser mujer. Barcelona: Anagrama.

Carrera, María Lina, Orrego-Hoyos, Gloria y Saralegui Ferrante, Natalia (2020). Dicen que tuve un bebé. Siete historias en las que el sistema judicial encarcela mujeres y a casi nadie le importa. Buenos Aires: Siglo XXI.

Favilli, Elena y Cavallo, Francesca (2016). Cuentos de buenas noches para niñas rebeldes. 100 historias de mujeres extraordinarias. Ciudad Autónoma de Buenos Aires: Planeta.

Galeano, Eduardo. Mujeres. Buenos Aires: Siglo XXI.

Grau, Didi (2018). Cereza y el río. Ciudad Autónoma de Buenos Aires: Edelvives.

Ibsen, Henrik ([1879], 2002). Casa de muñecas. Buenos Aires: Longseller.

Manzano, Eva y Gutiérrez Serna, Mónica (2015). Recetas de lluvia y azúcar. Barcelona: Thule Ediciones S.L.

Machado, Ana María y Calle, Carolina (2007). Punto a punto. Colombia: Babel.

Pronsky, Lorena (2018). Rota, se camina igual. Buenos Aires: Hojas del Sur. 
Walsh, María Elena (2018). Manuelita ¿Dónde vas? Ciudad Autónoma de Buenos Aires: Alfaguara.

Salvador Lavado, Joaquín, Quino (1993). Toda Mafalda. Buenos Aires: Ediciones de La Flor S.R.L.

Stanojevic, Jorge (2007). Cuentos y leyendas indígenas. Buenos Aires: Bonsái.

Turín, Adela y Bosnia, Nelia (1991). Rosa caramelo. Barcelona: Lumen, D.L.

Cuadernillo "Educación Sexual Integral para la Educación Primaria": http:// www.me.gov.ar/me_prog/esi/doc/esi_primaria.pdf

Especialización Docente de Nivel Superior en Educación y Derechos Humanos (2016). Educación y género: Clase 6: \#NiUnaMenos: El ámbito educativo como escenario de protección de derechos de las mujeres. Especialización en Derechos Humanos. Buenos Aires: Ministerio de Educación y Deportes de la Nación.

Especialización Docente de Nivel Superior en Educación y Derechos Humanos (2016). Educación y género: Clase 4: Educar/comunicar con una perspectiva de género. Especialización en Derechos Humanos. Buenos Aires: Ministerio de Educación y Deportes de la Nación.

Especialización Docente de Nivel Superior en Educación y Derechos Humanos (2016). Educación y género: Clase 5: Las masculinidades en debate. Especialización en Derechos Humanos. Buenos Aires: Ministerio de Educación y Deportes de la Nación.

Grimson, Alejandro (2015) "Los femicidios no son «cosas de mujeres»". Bala Colectivo Ni Una Menos. Página 12 "A un mes del 3 de junio".

Larralde, Gabriela (2014), Los mundos posibles. Disponible en https://es.scribd. com/document/241503869/Gabriela-Larralde-Los-mundos-posibles

Segato, Rita (2003) "Capítulo 4: La argamasa jerárquica: violencia moral, reproducción del mundo y la eficacia simbólica del Derecho." En Las Estructuras Elementales de la Violencia. Ensayos sobre género entre la antropología, el psicoanálisis y los derechos humanos. Quilmes, Argentina: Universidad Nacional de Quilmes Editorial.

Shock, Susy (2017). Crianzas. Historias para crecer en toda la diversidad. Argentina: Editorial muchas nueces.

Tufró, F y Huberman, H (2012) "4. Masculinidades", "5. Proceso de socialización de los jóvenes" $y$ "6. Estereotipos de género" en Modelo para armar. Nuevos desafíos de las masculinidades juveniles, pp. 12-20, de Asociación Civil TRAMA.

Para trabajar género con poesías, utilizaríamos textos de Gioconda Belli, Alejandra Pizarnik, Alfonsina Storni, Pedro Lemebel, Susy Shock, Episodios de 
La voz de Temis, obras de teatro y cortometraje "Aldana y León", entre otros.

\section{Títulos para trabajar con niñes}

Abella, Tomas (2008). M de mujer. Fundación Intermón Oxfam.

Bodoc, Liliana (2008). Amigos por el viento. Disponible en https://www. youtube.com/watch? $v=m W j \_U e T r X L U \& t=73 \mathrm{~s}$

Dourmec, Beatriz y Barnes, Ayax (2015). El pueblo que no quería ser gris. Buenos Aires: Ediciones Colihue.

Foreman, Michael (2009). El jardín feliz. Barcelona: Fundación Intermón Oxfam.

Katz, Karen (2008). Los colores de nuestra piel. Barcelona: Fundación Intermón Oxfam.

Gil, Carmen (2017). ¡Déjame en paz! Save the children.

Lipp, Frederick y Gaillard, Sofía (2007). Las zapatillas de Sofía. Fundación Intermón Oxfam.

Monfort, Mireia (2016). El poder de una sonrisa. La Asociación Española Síndrome de Phelan-McDermid.

Montes, Graciela (1985). La familia de la soga. Ediciones Colihue. Disponible en https://www.youtube.com/watch?v=0PUdNJfOSwM

Wernike, María (2012). Hay días. Buenos Aires: Calibroscopio.

Wernike, María (2013). Papá y yo a veces. Buenos Aires: Calibroscopio.

Wernike, María (2016). Cuando estamos juntas. Buenos Aires: Calibroscopio.

\section{Diversidad familiar}

Richardson, Judith y Peter Parnell (2005). Tres con tango. Estados Unidos: Simon \& Schuster. Trad: Francesc Strino. Disponible en https://www.youtube. com/watch?v=JLteRP7s2Jw

Linda De Hara y Stern Nijland. Rey y rey. Disponible en http://www. pakapaka.gob.ar/videos/132642

Mar Cerdá. Familiario. Disponible en https://www.youtube.com/watch?$\mathrm{v}=\mathrm{PmG} 2 \mathrm{VEXCGOk}$

Martino, Teresa M., Bauger, Adelina P., Ruesta, Francisco (2016). Divercuentos: cuentos infantiles sobre diversidad familiar. Contribuciones de Facundo Díaz Castellano... [et al.]. Dirigido por Cecilia López. -1ª ed.- La Plata: Universidad Nacional de La Plata. Facultad de Ciencias Jurídicas y Sociales. 